\title{
Piecewise Cubic Interpolation and Two-Point Boundary Value Problems
}

\author{
By \\ Manabu SAKAI*
}

\section{Introduction}

Cubic splines are of much use for approximating solutions of simple two-point boundary value problems for both linear and nonlinear ordinary differential equations. In the present paper, we shall give its mathematical foundation by the use of Urabe's method [5], which is quite universally applicable.

We consider the following two-point boundary value problem:

$$
\ddot{x}(t)=f(t, x(t), \dot{x}(t)) \quad(0 \leq t \leq 1)
$$

with boundary conditions

$$
\begin{aligned}
& A_{0} x(0)-B_{0} \dot{x}(0)=a \\
& A_{1} x(1)+B_{1} \dot{x}(1)=b
\end{aligned}
$$

where $f(t, x, w)$ is defined and twice continuously differentiable in a region $D$ of $(t, x, w)$-space intercepted by two hyperplanes $t=0$ and $t=1$.

We rewrite the problem (1)-(3) in the following form:

$$
\dot{x}(t)=w(t)
$$$$
(0 \leq t \leq 1)
$$

$$
\dot{w}(t)=f(t, x(t), w(t))
$$

Received March 24, 1971.

Communicated by M. Urabe.

* Department of Mathematics, Fukuoka University, Fukuoka, Japan. 


$$
A_{1} x(1)+B_{1} w(1)=b
$$

Now making use of $B$-spline $Q_{m+1}(t)=\frac{1}{m !} \sum_{i=0}^{m+1}(-1)^{i}\left({ }_{i}^{m+1}\right)(t-i)_{+}^{m}$, we consider spline functions of the form $x_{h}(t)=\sum_{p=-3}^{n-1} \alpha_{p} Q_{4}\left(\frac{t}{h}-p\right)$, $w_{h}(t)=\sum_{p=-2}^{n-1} \beta_{p} Q_{3}\left(\frac{t}{h}-p\right)(n h=1)$ with undetermined coefficients $\alpha_{-3}$, $\alpha_{-2}, \ldots, \alpha_{n-1}$ and $\beta_{-2}, \beta_{-1}, \ldots, \beta_{n-1}$. The above $x_{h}$ and $w_{h}$ will be an approximate solution to the problem (4)-(7) if they satisfy

$$
\begin{array}{ll}
\dot{x}_{h}(t)=w_{h}(t) & (0 \leq t \leq 1) \\
\dot{w}_{h}(t)=P f\left(t, x_{h}(t), w_{h}(t)\right) & (0 \leq t \leq 1) \\
A_{0} x_{h}(0)-B_{0} w_{h}(0)=a & \\
A_{1} x_{h}(1)+B_{1} w_{h}(1)=b . &
\end{array}
$$

Here $P$ is an operator defined by $(P f)(t)=\sum_{p=0}^{n} f\left(t_{p}\right) L_{p}(t)$, where $L_{p}(t)$ is a piecewise linear function with property $L_{p}\left(t_{q}\right)=\delta_{p, q}\left(t_{q}=q h\right)$. From a well known relation $\dot{Q}_{m+1}(t)=Q_{m}(t)-Q_{m}(t-1)$, we see that equation (8) is equivalent to the following system of $n+2$ equations:

$$
\frac{\alpha_{p}-\alpha_{p-1}}{h}=\beta_{p} \quad(p=-2,-1, \ldots, n-1)
$$

Any two piecewise linear functions coincide with each other if and only if they coincide at the nodes, therefore we see that equation (9) is equivalent to the following system of $n+1$ equations:

$$
\begin{array}{r}
\frac{\beta_{p-1}-\beta_{p-2}}{h}=f\left(t_{p}, \frac{\alpha_{p-3}+4 \alpha_{p-2}+\alpha_{p-1}}{6}, \frac{\beta_{p-2}+\beta_{p-1}}{2}\right) \\
\quad(p=0,1, \ldots, n) .
\end{array}
$$

The boundary conditions (10) and (11) give two equations:

$$
A_{0} \frac{\alpha_{-3}+4 \alpha_{-2}+\alpha_{-1}}{6}-B_{0} \frac{\beta_{-2}+\beta_{-1}}{2}=a
$$




$$
A_{1} \frac{\alpha_{n-3}+4 \alpha_{n-2}+\alpha_{n-1}}{6}+B_{1} \frac{\beta_{n-2}+\beta_{n-1}}{2}=b
$$

The number of undetermined coefficients is $2 n+5$ and the conditions (12)-(15) precisely give the requisite number of equations. For the convenience of the analysis, we rewrite (12)-(15) in the following form:

$$
\begin{aligned}
F_{h}^{(1)}(\alpha, \beta)= & \left\{\frac{\alpha_{p}-\alpha_{p-1}}{h}-\beta_{p}\right\}_{p=-2}^{n-1}=0, \\
F_{h}^{(2)}(\alpha, \beta)= & \frac{\beta_{p-1}-\beta_{p-2}}{h}-f\left(t_{p}, \frac{\alpha_{p-3}+4 \alpha_{p-2}+\alpha_{p-1}}{6},\right. \\
& \left.\left.\frac{\beta_{p-2}+\beta_{p-1}}{2}\right)\right\}_{p=0}^{n}=0, \\
F_{h}^{(3)}(\alpha, \beta)= & \left\{A_{0} \frac{\alpha_{-3}+4 \alpha_{-2}+\alpha_{-1}}{6 \sqrt{h}}-B_{0} \frac{\beta_{-2}+\beta_{-1}}{2 \sqrt{h}}-\frac{a}{\sqrt{h}},\right. \\
& \left.A_{1} \frac{\alpha_{n-3}+4 \alpha_{n-2}+\alpha_{n-1}}{6 \sqrt{h}}+B_{1} \frac{\beta_{n-2}+\beta_{n-1}}{2 \sqrt{h}}-\frac{b}{\sqrt{h}}\right\}=0 .
\end{aligned}
$$

In what follows, the system of equations (16) will be called a determining equation for spline approximations. In the present paper we use (16) in order to facilitate the analysis, but clearly in practical computations it is more convenient to use the equations containing only $\alpha_{p}(p=-3,-2, \ldots$, $n-1)$ which can be obtained from $(12)-(15)$ by eliminating $\beta_{p}$.

In the present paper we assume that the problem (4)-(7) has an isolated solution $(\hat{x}(t), \hat{w}(t))$ satisfying the internality condition

$$
U=\left\{(t, x, w) \mid\left[(x-\hat{x}(t))^{2}+(w-\hat{w}(t))^{2}\right]^{\frac{1}{2}} \leq \delta, t \in[0,1]\right\} \subset D
$$

for some $\delta>0$. By the definition in $[4]$ (p. 46), a solution $(\hat{x}(t), \hat{w}(t))$ to the problem (4)-(7) is isolated if and only if

$$
G=\left[\begin{array}{ll}
A_{0} & -B_{0} \\
A_{1} y_{1}(1)+B_{1} z_{1}(1) & A_{1} y_{2}(1)+B_{1} z_{2}(1)
\end{array}\right]
$$

is non-singular, where 


$$
\left[\begin{array}{ll}
y_{1}(t) & y_{2}(t) \\
z_{1}(t) & z_{2}(t)
\end{array}\right]=\Phi(t)
$$

is a fundamental matrix with property $\Phi(0)=E$ ( $E$ the unit matrix) of the first variation equation of (4)-(5) with respect to $(\hat{x}(t), \hat{w}(t))$, that is,

$$
\begin{gathered}
\dot{y}(t)=z(t), \\
\dot{z}(t)=f_{x}(t, \hat{x}(t), \hat{w}(t)) y(t)+f_{w}(t, \hat{x}(t), \hat{w}(t)) z(t) .
\end{gathered}
$$

Corresponding to $\hat{x}(t)$, as easily seen, one can determine uniquely a cubic spline function $\hat{x}_{h}(t)$ of the form

$$
\hat{x}_{h}(t)=\sum_{p=-3}^{n-1} \hat{\alpha}_{p} Q_{4}\left(\frac{t}{h}-p\right)
$$

so that

$$
\begin{aligned}
& \hat{x}_{h}\left(t_{p}\right)=\hat{x}\left(t_{p}\right) \quad(p=0,1,2, \ldots, n), \\
& \dot{\hat{x}}_{h}\left(t_{0}\right)=\dot{\hat{x}}\left(t_{0}\right), \\
& \dot{\hat{x}}_{h}\left(t_{n}\right)=\dot{\hat{x}}\left(t_{n}\right) .
\end{aligned}
$$

Since $\hat{x}(t) \in C^{4}[0,1]$ due to the assumption $f(t, x, w) \in C_{t, x, w}^{2}(D)$, by Theorem 2.3.4 in [1] (p. 29) it is valid that

$$
\left|\left(\frac{d}{d t}\right)^{k}\left[\hat{x}_{h}(t)-\hat{x}(t)\right]\right|=O\left(h^{4-k}\right) \quad(k=0,1,2,3)
$$

uniformly on $[0,1]$ as $h \rightarrow 0$. From $\hat{x}_{h}(t)$, one can easily construct a quadratic spline function $\hat{w}_{h}(t)$ of the form

$$
\hat{w}_{h}(t)=\sum_{p=-2}^{n-1} \hat{\beta}_{p} Q_{3}\left(\frac{t}{h}-p\right)
$$

so that

$$
\hat{w}_{h}(t)=\dot{\hat{x}}_{h}(t) \quad(0 \leq t \leq 1) .
$$


By (20), it is then valid that

$$
\begin{aligned}
& \left|\hat{w}_{h}(t)-\hat{w}(t)\right|=\left|\dot{\hat{x}}_{h}(t)-\dot{\hat{x}}(t)\right|=O\left(h^{3}\right) \\
& \left|\dot{\hat{w}}_{h}(t)-\dot{\hat{w}}(t)\right|=\left|\ddot{\hat{x}}_{h}(t)-\ddot{\hat{x}}(t)\right|=O\left(h^{2}\right)
\end{aligned}
$$

uniformly on $[0,1]$ as $h \rightarrow 0$.

\section{Some Properties of Spline Functions}

In what follows, for any $\varphi(t) \in L^{2}[0,1]$, we shall denote $\left[\int_{0}^{1} \varphi^{2}(t) d t\right]^{\frac{1}{2}}$ by $\|\varphi\|$, and for any finite dimensional vector $c$, we shall denote its Euclidean norm by $|c|$.

Lemma 1. Let $\varphi(t)=\sum_{0=p}^{n} c_{p} L_{p}(t)$, then $\mu_{1} \sqrt{h}|c| \leq\|\varphi\| \leq \lambda_{1} \sqrt{h}|c|$, where $\mu_{1}$ and $\lambda_{1}$ are positive constants independent of $h$, and $c=\left(c_{0}\right.$, $\left.c_{1}, c_{2}, \ldots, c_{n}\right)$.

Proof. For $t \in\left[t_{p}, t_{p+1}\right](p=0,1, \ldots, n-1), \varphi(t)=c_{p} L_{p}(t)+c_{p+1} L_{p+1}(t)$. Therefore we easily get

$$
\|\varphi\|^{2}=\sum_{p=0}^{n-1} \int_{t_{p}}^{t_{p+1}} \varphi^{2}(t) d t=\frac{h}{3} \sum_{p=0}^{n-1}\left(c_{p}^{2}+c_{p} c_{p+1}+c_{p+1}^{2}\right) .
$$

Since $\frac{1}{2}\left(c_{p}^{2}+c_{p+1}^{2}\right) \leq c_{p}^{2}+c_{p} c_{p+1}+c_{p+1}^{2} \leq \frac{3}{2}\left(c_{p}^{2}+c_{p+1}^{2}\right)$, we have $\frac{1}{6} h|c|^{2}$ $\leq\|\varphi\|^{2} \leq h|c|^{2}$, which proves the lemma with $\mu_{1}=\frac{1}{\sqrt{6}}$ and $\lambda_{1}=1$.

Lemma 2. Let $\varphi(t)=\sum_{p=-1}^{n-1} c_{p} Q_{3}\left(\frac{t}{h}-p\right), \quad$ then $\quad \mu_{2} \sqrt{h}|c| \leq\|\varphi\| \leq$ $\lambda_{2} \sqrt{h}|c|$, where $\mu_{2}$ and $\lambda_{2}$ are positive constants independent of $h$, and $c=\left(c_{-2}, c_{-1}, \ldots, c_{n-1}\right)$.

Proof. Since $\varphi(t)$ is a quadratic polynomial on $\left[t_{p}, t_{p+1}\right](p=0,1$, $2, \ldots, n-1)$, it can be written on $\left[t_{p}, t_{p+1}\right]$ as follows:

$$
\varphi(t)=\varphi\left(t_{p}\right)+\dot{\varphi}\left(t_{p}\right)\left(t-t_{p}\right)+\frac{\ddot{\varphi}\left(t_{p}+\right)}{2 !}\left(t-t_{p}\right)^{2}
$$

Here it is easily seen that $\varphi\left(t_{p}\right)=\frac{c_{p-2}+c_{p-1}}{2}, \dot{\varphi}\left(t_{p}\right)=\frac{c_{p-1}-c_{p-2}}{h}$, and 
$\ddot{\varphi}\left(t_{p}+\right)=\frac{c_{p-2}-2 c_{p-1}+c_{p}}{h^{2}}$. Therefore it follows that

$$
\begin{aligned}
\|\varphi\|^{2} & =\sum_{p=0}^{n-1} \int_{t_{p}}^{t_{p+1}} \varphi^{2}(t) d t \\
& \left.=\sum_{p=0}^{n-1} h \int_{0}^{1}\left(\varphi\left(t_{p}\right)+h \dot{\varphi}\left(t_{p}\right) t+\frac{h^{2}}{2} \ddot{\varphi}\left(t_{p}+\right) t^{2}\right)\right)^{2} d t \\
& =\sum_{p=0}^{n-1} h I_{p},
\end{aligned}
$$

where $I_{p}$ is a quadratic form with respect to $c_{p-2}, c_{p-1}$ and $c_{p}$ independent of $h . I_{p}$ is nonnegative, and $I_{p}=0$ implies that $\varphi\left(t_{p}\right)=\dot{\varphi}\left(t_{p}\right)=\ddot{\varphi}\left(t_{p}+\right)=0$, that is, $c_{p-2}=c_{p-1}=c_{p}=0$. Therefore $I_{p}$ is a positive definite quadratic form, consequently there are positive constants $\mu_{2}$ and $\lambda_{2}$ such that $\mu_{2}^{2}\left(c_{p-2}^{2}\right.$ $\left.+c_{p-1}^{2}+c_{p}^{2}\right) \leq I_{p} \leq \frac{\lambda_{2}^{2}}{3}\left(c_{p-2}^{2}+c_{p-1}^{2}+c_{p}^{2}\right)$. From this readily follows the conclusion of the lemma.

Lemma 3. Let $\varphi(t)=\sum_{p=-3}^{n-1} c_{p} Q_{4}\left(\frac{t}{h}-p\right), \quad$ then $\quad \mu_{3} \sqrt{h}|c| \leq\|\varphi\| \leq$ $\lambda_{3} \sqrt{h}|c|$, where $\mu_{3}$ and $\lambda_{3}$ are positive constants independent of $h$, and $c=\left(c_{-3}, c_{-2} \ldots, c_{n-1}\right)$.

Proof. Since $\varphi(t)$ is a cubic polynomial on $\left[t_{p}, t_{p+1}\right](p=0,1, \ldots$, $n-1)$, it can be written on $\left[t_{p}, t_{p+1}\right]$ as follows:

$$
\varphi(t)=\varphi\left(t_{p}\right)+\dot{\varphi}\left(t_{p}\right)\left(t-t_{p}\right)+\frac{\ddot{\varphi}\left(t_{p}\right)}{2 !}\left(t-t_{p}\right)^{2}+\frac{\dddot{\varphi}\left(t_{p}+\right)}{3 !}\left(t-t_{p}\right)^{3} .
$$

Here it is easily seen that $\varphi\left(t_{p}\right)=\frac{c_{p-3}+4 c_{p-2}+c_{p-1}}{6}, \dot{\varphi}\left(t_{p}\right)=\frac{c_{p-1}-c_{p-3}}{2 h}$, $\ddot{\varphi}\left(t_{p}\right)=\frac{c_{p-1}-2 c_{p-2}+c_{p-3}}{h^{2}}$, and $\dddot{\varphi}\left(t_{p}+\right)=\frac{c_{p-1}-3 c_{p-1}+3 c_{p-2}-c_{p-3}}{h^{3}}$. Hence likewise as the proof of lemma 2, we have easily the conclusion of the present lemma.

Lemma 4. Let $\varphi(t)=\sum_{p=-m}^{n-1} c_{p} Q_{m+1}\left(\frac{t}{h}-p\right)$, then $\|\varphi\|_{\infty} \leq|c|_{\infty}$ for $m \geq 1$, where $\|\varphi\|_{\infty}=\sup _{0 \leq t \leq 1}|\varphi(t)|, c=\left(c_{-m}, \cdots, c_{n-1}\right)$ and $|c|_{\infty}=\max _{p}\left|c_{p}\right|$. 
Proof. As well known, $B$-splines can be characterized by convolution of characteristic functions as follows:

$$
Q_{m+1}(t)=(\underbrace{\chi * \cdots * x}_{m+1})(t),
$$

where

$$
\chi(t)=\left\{\begin{array}{l}
1 \text { for } t \in[0,1] \\
0 \text { for } t \notin[0,1] .
\end{array}\right.
$$

From this characterization of $B$-splines it readily follows that
(i) $0 \leq Q_{m+1}(t) \leq 1$
$(m=0,1,2, \cdots)$
(ii) $\sum_{p=-\infty}^{\infty} Q_{m+1}(t-p)=1$
$(m=1,2, \ldots)$.

From these inequalities, we readily get the desired inequality.

Lemma 5. If $g(t)$ is continuously differentiable, then

$$
\left.\|(I-p) g\| \leq \frac{h}{\sqrt{6}}\|\dot{g}\| \quad \text { (I the unit operator }\right) .
$$

Proof. One can easily verify that

$$
(I-p) g(t)=\int_{t_{p}}^{t_{p+1}} k_{p}(t, s) \dot{g}(s) d s \quad \text { for any } t \in\left[t_{p}, t_{p+1}\right],
$$

where $k_{p}(t, s)$ is a piecewise continuous function such that

$$
k_{p}(t, s)=\left\{\begin{array}{cl}
1-\frac{t-t_{p}}{h} & \text { if } s \leq t, \\
-\frac{t-t_{p}}{h} & \text { if } t<s .
\end{array}\right.
$$

One then has

$$
\begin{aligned}
\int_{t_{p}}^{t_{p+1}}[(I-p) g(t)]^{2} d t & \leq \int_{t_{p}}^{t_{p+1}} d t \int_{t_{p}}^{t_{p+1}} k_{p}^{2}(t, s) d s \int_{t_{p}}^{t_{p+1}} \dot{g}^{2}(s) d s \\
& \leq \frac{h^{2}}{6} \int_{t_{p}}^{t_{p+1}} \dot{g}^{2}(s) d s
\end{aligned}
$$


from which readily follows the conclusion of the lemma.

3. The Jacobian Matrix of $\left(F_{h}^{(1)}(\alpha, \beta), F_{h}^{(2)}(\alpha, \beta), F_{h}^{(3)}(\alpha, \beta)\right)$

Put $\left(F_{h}^{(1)}(\alpha, \beta), F_{h}^{(2)}(\alpha, \beta), F_{h}^{(3)}(\alpha, \beta)\right)=F_{h}(\alpha, \beta)$ and let $J_{h}(\alpha, \beta)$ be the Jacobian matrix of $F_{h}(\alpha, \beta)$ with respect to $\alpha_{p}(p=-3,-2 \ldots, n-1)$ and $\beta_{p}(p=-2,-1, \ldots, n-1)$. In order to investigate the properties of $J_{h}(\alpha, \beta)$, let us consider a linear system

$$
J_{h}(\alpha, \beta)\left(\xi_{1}, \xi_{2}\right)+\left(\gamma_{1}, \gamma_{2}, \gamma_{3}\right)=0
$$

where $\xi_{1}=\left(u_{-3}, u_{-2}, \ldots, u_{n-1}\right), \xi_{2}=\left(v_{-2}, v_{-1}, \ldots, v_{n-1}\right), \gamma_{1}=\left(c_{-2}, c_{-1}, \ldots\right.$, $\left.c_{n-1}\right), \gamma_{2}=\left(d_{0}, d_{1}, \ldots, d_{n-1}\right)$ and $\gamma_{3}=\left(e_{1}, e_{2}\right)$.

Corresponaing to $\xi_{1}$ and $\xi_{2}$, we consider cubic and quadratic spline functions $y_{1}(t)$ and $y_{2}(t)$ defined by

$$
y_{1}(t)=\sum_{p=-3}^{n-1} u_{p} Q_{4}\left(\frac{t}{h}-p\right) \text { and } y_{2}(t)=\sum_{p=-2}^{n-1} v_{p} Q_{3}\left(\frac{t}{h}-p\right)
$$

and in a similar way, corresponding to $\gamma_{1}$ and $\gamma_{2}$, we consider quadratic and linear spline functions $\varphi_{1}(t)$ and $\varphi_{2}(t)$ defined by

$$
\varphi_{1}(t)=\sum_{p=-2}^{n-1} c_{p} Q_{3}\left(\frac{t}{h}-p\right) \text { and } \varphi_{2}(t)=\sum_{p=0}^{n} d_{p} L_{p}(t)
$$

Since $J(\alpha, \beta)\left(\xi_{1} \xi_{2}\right)=\lim _{\theta \rightarrow 0} \theta^{-1}\left[F\left(\alpha+\theta \xi_{1}, \beta+\theta \xi_{2}\right)-F(\alpha, \beta)\right]$, corresponding to (22), we have:

$$
\begin{aligned}
& \text { (23) } \begin{aligned}
\dot{y}_{1}(t) & =y_{2}(t)-\varphi_{1}(t) \quad(0 \leq t \leq 1), \\
\text { (24) } \quad \dot{y}_{2}\left(t_{p}\right) & =f_{x}\left(t_{p}, \frac{\alpha_{p-3}+4 \alpha_{p-2}+\alpha_{p-1}}{6}, \frac{\beta_{p-2}+\beta_{p-1}}{2}\right) y_{1}\left(t_{p}\right) \\
& +f_{w}\left(t_{p}, \frac{\alpha_{p-3}+4 \alpha_{p-2}+\alpha_{p-1}}{6}, \frac{\beta_{p-2}+\beta_{p-1}}{2}\right) y_{2}\left(t_{p}\right) \\
& -\varphi_{2}\left(t_{p}\right) \quad(p=0,1, \ldots, n)
\end{aligned} \\
& \text { (25) } \quad A_{0} y_{1}(0)-B_{0} y_{2}(0)=-\sqrt{h} e_{1}, \\
& \text { (26) } A_{1} y_{1}(1)+B_{1} y_{2}(1)=-\sqrt{h} e_{2} .
\end{aligned}
$$


Now substitute $(\hat{\alpha}, \hat{\beta})$ for $(\alpha, \beta)$ in $(24)$, then we have

$$
\begin{array}{r}
\dot{y}_{2}(t)=P\left[f_{x}\left(t, \hat{x}_{h}(t), \hat{w}_{h}(t)\right) y_{1}(t)+f_{w}\left(t, \hat{x}_{h}(t), \hat{w}_{h}(t)\right) y_{2}(t)\right]-\varphi_{2}(t) \\
(0 \leq t \leq 1),
\end{array}
$$

since $\dot{y}_{2}(t)$ and $\varphi_{2}(t)$ are both piecewise linear. Equation (24) can be rewritten as follows:

$$
\dot{y}_{2}(t)=f_{x}\left(t, \hat{x}_{h}(t), \hat{w}_{h}(t)\right) y_{1}(t)+f_{w}\left(t, \hat{x}_{h}(t), \hat{w}_{h}(t)\right) y_{2}(t)+R(t)-\varphi_{2}(t)
$$

where $R=-(I-P)\left[f_{x}\left(t, \hat{x}_{h}(t), \hat{w}_{h}(t)\right) y_{1}(t)+f_{w}\left(t, \hat{x}_{h}(t), \hat{w}_{h}(t)\right) y_{2}(t)\right], \quad(I$ the unit operator).

Now by (23) and (27) we have:

$$
\begin{aligned}
& \frac{d}{d t}\left[f_{x}\left(t, \hat{x}_{h}(t), \hat{w}_{h}(t)\right) y_{1}(t)+f_{w}\left(t, \hat{x}_{h}(t), \hat{w}_{h}(t)\right) y_{2}(t)\right] \\
= & f_{x}\left(t, \hat{x}_{h}(t), \hat{w}_{h}(t)\right)\left(y_{2}(t)-\varphi_{1}(t)\right)+f_{w}\left(t, \hat{x}_{h}(t), \hat{w}_{h}(t)\right) \\
& \times\left[f_{x}\left(t, \hat{x}_{h}(t), \hat{w}_{h}(t)\right) \cdot y_{1}(t)+f_{w}\left(t, \hat{x}_{h}(t), \hat{w}_{h}(t)\right) y_{2}(t)+R(t)-\varphi_{2}(t)\right] \\
& +\frac{d}{d t} f_{x}\left(t, \hat{x}_{h}(t), \hat{w}_{h}(t)\right) \cdot y_{1}(t)+\frac{d}{d t} f_{w}\left(t, \hat{x}_{h}(t), \hat{w}_{h}(t)\right) \cdot y_{2}(t)
\end{aligned}
$$

for any $t \in[0,1]$. On the other hand, if $h_{0}$ is sufficiently small, by (20) and (21) there is $K_{1}>0$ such that

$$
\begin{aligned}
& \left|f_{x}\left(t, \hat{x}_{h}(t), \hat{w}_{h}(t)\right)\right|,\left|f_{w}\left(t, \hat{x}_{h}(t), \hat{w}_{h}(t)\right)\right|,\left|\frac{d}{d t} f_{x}\left(t, \hat{x}_{h}(t), \hat{w}_{h}(t)\right)\right|, \\
& \left|\frac{d}{d t} f_{w}\left(t, \hat{x}_{h}(t), \hat{w}_{h}(t)\right)\right| \leq K_{1} \quad(0 \leq t \leq 1) \quad \text { for any } h \leq h_{0} .
\end{aligned}
$$

Therefore by Lemma 5 we have

$$
\begin{aligned}
\|R\| \leq \frac{h}{\sqrt{6}}\left[K_{1}\left(\left\|y_{2}\right\|+\left\|\varphi_{1}\right\|\right)+K_{1}\left\{K_{1}\left(\left\|y_{1}\right\|+\left\|y_{2}\right\|\right)+\|R\|+\left\|\varphi_{2}\right\|\right\}\right. \\
\left.+K_{1}\left(\left\|y_{1}\right\|+\left\|y_{2}\right\|\right)\right]
\end{aligned}
$$


from which follows

$$
\left(1-\frac{h}{\sqrt{6}} K_{1}\right)\|R\| \leq \frac{h}{\sqrt{6}} K_{1}\left[\left(1+K_{1}\right)\left\|y_{1}\right\|+\left(2+K_{1}\right)\left\|y_{2}\right\|+\left\|\varphi_{1}\right\|+\left\|\varphi_{2}\right\|\right]
$$

Hence we have the estimate of $\|R\|$ of the form

$$
\|R\| \leq \frac{h}{\sqrt{2}} K\left[\left(\left\|y_{1}\right\|+\left\|y_{2}\right\|\right)+\left(\left\|\varphi_{1}\right\|+\left\|\varphi_{2}\right\|\right)\right]
$$

or

$$
\|R\| \leqq h K\left[\left\|\left(y_{1}, y_{2}\right)\right\|+\left\|\left(\varphi_{1}, \varphi_{2}\right)\right\|\right]
$$

where

$$
\begin{aligned}
& \left\|\left(y_{1}, y_{2}\right)\right\|=\left[\int_{0}^{1}\left|\left(y_{1}(t), y_{2}(t)\right)\right|^{2} d t\right]^{\frac{1}{2}}=\left[\left\|y_{1}\right\|^{2}+\left\|y_{2}\right\|^{2}\right]^{\frac{1}{2}}, \\
& \left\|\left(\varphi_{1}, \varphi_{2}\right)\right\|=\left[\int_{0}^{1}\left|\left(\varphi_{1}(t), \varphi_{2}(t)\right)\right|^{2} d t\right]^{\frac{1}{2}}=\left[\left\|\varphi_{1}\right\|^{2}+\left\|\varphi_{2}\right\|^{2}\right]^{\frac{1}{2}} .
\end{aligned}
$$

Now let $\Phi_{h}(t)$ be the fundamental matrix with property $\Phi_{h}(0)=E$ of the following linear homogeneous system:

$$
\begin{aligned}
& \dot{y}(t)=z(t) \\
& \dot{z}(t)=f_{x}\left(t, \hat{x}_{h}(t), \hat{u}_{h}(t)\right) y(t)+f_{w}\left(t, \hat{x}_{h}(t), \hat{w}_{h}(t)\right) z(t),
\end{aligned}
$$

then comparing (29) with (18), by (20) and (21), we have

$$
\left|\Phi_{h}(t)-\Phi(t)\right|=O\left(h^{3}\right) \quad(0 \leq t \leq 1),
$$

therefore we have a non-singular matrix $G_{h}$ associated with (29) corresponding to $G$ associated with (18) by (17). Then applying Proposition 1 of $[4]$ (p. 44) to the system of equations (23), (27), (25) and (26), we have

$$
\left(\begin{array}{l}
y_{1}(t) \\
y_{2}(t)
\end{array}\right)=-\Phi_{h}(t) G_{h}^{-1}\left(\begin{array}{c}
\sqrt{h} e_{1} \\
\sqrt{h} e_{2}
\end{array}\right)+\int_{0}^{1} H_{h}(t, s)\left(\begin{array}{c}
-\varphi_{1}(s) \\
R(s)-\varphi_{2}(s)
\end{array}\right) d s
$$


where

(32) $H_{h}(t, s)= \begin{cases}\Phi_{h}(t)\left[E-G_{h}^{-1}\left(\begin{array}{ll}0 & 0 \\ A_{1} & B_{1}\end{array}\right) \Phi_{h}(1)\right] \Phi_{h}^{-1}(s) & (s \leq t) \\ -\Phi_{h}(t) G_{h}^{-1}\left(\begin{array}{cc}0 & 0 \\ A_{1} & B_{1}\end{array}\right) \Phi_{h}(1) \Phi_{h}^{-1}(s) & (t<s) .\end{cases}$

From (31), by (30) we have the inequality of the form

$$
\left\|\left(y_{1}, y_{2}\right)\right\| \leq \sqrt{h} M_{1}\left|\left(e_{1}, e_{2}\right)\right|+M_{2} \|\left(-\varphi_{1}, R-\varphi_{2}\right) i \mid
$$

for any $h \leq h_{0}$ provided $h_{0}$ is sufficiently small. Here $M_{1}$ and $M_{2}$ are positive constants independent of $h$. Since

$$
\left\|\left(-\varphi_{1}, R-\varphi_{2}\right)\right\| \leq\|R\|+\left\|\varphi_{1}\right\|+\left\|\varphi_{2}\right\| \leq\|R\|+\sqrt{2}\left\|\left(\varphi_{1}, \varphi_{2}\right)\right\|,
$$

by the use of (28) we then have

$$
\left(1-h K M_{2}\right)\left\|\left(y_{1}, y_{2}\right)\right\| \leq \sqrt{h} M_{1}\left|\left(e_{1}, e_{2}\right)\right|+M_{2}(\sqrt{2}+h K)\left\|\left(\varphi_{1}, \varphi_{2}\right)\right\|,
$$

from which we obtain the inequality of the form

$$
\left\|\left(y_{1}, y_{2}\right)\right\| \leq \sqrt{h} M_{3}\left|\left(e_{1}, e_{2}\right)\right|+M_{4}||\left(\varphi_{1}, \varphi_{2}\right) \|
$$

for any $h \leq h_{0}$ provided $h_{0}$ is sufficiently small. Now by Lemmas 1,2 and 3 ,

$$
\begin{aligned}
& \left|\xi_{1}\right| \leq \frac{1}{\mu_{3}} \cdot \frac{1}{\sqrt{h}}\left\|y_{1}\right\|, \quad\left|\xi_{2}\right| \leq \frac{1}{\mu_{2}} \cdot \frac{1}{\sqrt{h}}\left\|y_{2}\right\|, \\
& \left\|\varphi_{1}\right\| \leq \lambda_{2} \sqrt{h}\left|\gamma_{1}\right|, \quad\left\|\varphi_{2}\right\| \leq \lambda_{1} \sqrt{h}\left|\gamma_{2}\right| .
\end{aligned}
$$

Therefore we finally have the inequality of the form

$$
\left|\left(\xi_{1}, \xi_{2}\right)\right| \leq M\left|\left(\gamma_{1}, \gamma_{2}, \gamma_{3}\right)\right|
$$

for any $h \leq h_{0}$ provided $h_{0}$ is sufficiently small. Here $M$ is a positive constant independent of $h$. By (22), inequality (33) implies the nonsingularity of $J_{h}(\hat{\alpha}, \hat{\beta})$ and in addition the inequality

$$
\left|J_{h}^{-1}(\hat{\alpha}, \hat{\beta})\right| \leq M \quad \text { for any } h \leq h_{0} .
$$


Let $\alpha=\left(\alpha_{-3}, \alpha_{-2}, \cdots, \alpha_{n-1}\right), \beta=\left(\beta_{-2}, \beta_{-1}, \cdots, \beta_{n-1}\right), \alpha^{\prime}=\left(\alpha_{-3}^{\prime}, \alpha_{-2}^{\prime}, \cdots\right.$ $\left.\alpha_{n-1}^{\prime},\right), \beta^{\prime}=\left(\beta_{-2}^{\prime}, \beta_{-1}^{\prime}, \cdots, \beta_{n-1}^{\prime}\right)$ be arbitrary vectors that

$$
\begin{array}{r}
\left\{\left[\frac{1}{6}\left(\alpha_{p-3}+4 \alpha_{p-2}+\alpha_{p-1}\right)-\hat{x}\left(t_{p}\right)\right]^{2}+\left[\frac{1}{2}\left(\beta_{p-2}+\beta_{p-1}\right)-\hat{w}\left(t_{p}\right)\right]^{2}\right\}^{\frac{1}{2}} \leq \delta \\
\left\{\left[\frac{1}{6}\left(\alpha_{p-3}^{\prime}+4 \alpha_{p-2}^{\prime}+\alpha_{p-1}^{\prime}\right)-\hat{x}\left(t_{p}\right)\right]^{2}+\left[\frac{1}{2}\left(\beta_{p-2}^{\prime}+\beta_{p-1}^{\prime}\right)-\hat{w}\left(t_{p}\right)\right]^{2}\right\}^{\frac{1}{2}} \leq \delta \\
(p=0,1, \ldots, n) .
\end{array}
$$

Put

$$
\begin{aligned}
& \frac{\partial}{\partial \alpha_{q_{1}}} f\left[t_{p}, \frac{1}{6}\left(\alpha_{p-3}+4 \alpha_{p-2}+\alpha_{p-1}\right), \frac{1}{2}\left(\beta_{p-2}+\beta_{p-1}\right)\right]=J_{p q_{1}}^{(2)}(\alpha, \beta), \\
& \frac{\partial}{\partial \beta_{q_{2}}} f\left[t_{p}, \frac{1}{6}\left(\alpha_{p-3}+4 \alpha_{p-2}+\alpha_{p-1}\right), \frac{1}{2}\left(\beta_{p-2}+\beta_{p-1}\right)\right]=J_{p q_{2}}^{(2)}(\alpha, \beta),
\end{aligned}
$$

then from (16) it is easily seen that

$$
\begin{aligned}
& \left|J_{h}(\alpha, \beta)-J_{h}\left(\alpha^{\prime}, \beta^{\prime}\right)\right|^{2} \leq \sum_{p=0}^{n}\left\{\sum_{q_{1}}\left[J_{p q_{1}}^{(2)}(\alpha, \beta)-J_{p q_{1}}^{(2)}\left(\alpha^{\prime}, \beta^{\prime}\right)\right]^{2}\right. \\
& \left.\quad+\sum_{q_{2}}\left[J_{p q_{2}}^{(2)}(\alpha, \beta)-J_{p q_{2}}^{(2)}\left(\alpha^{\prime}, \beta^{\prime}\right)\right]^{2}\right\} .
\end{aligned}
$$

Now

$$
\begin{aligned}
& J_{p q_{1}}^{(2)}(\alpha, \beta)= f_{x}\left[t_{p}, \frac{1}{6}\left(\alpha_{p-3}+4 \alpha_{p-2}+\alpha_{p-1}\right), \frac{1}{2}\left(\beta_{p-2}+\beta_{p-1}\right)\right] . \\
& \frac{1}{6}\left(\delta_{q_{1}, p-3}+4 \delta_{q_{1}, p-2}+\delta_{q_{1}, p-1}\right), \\
& J_{p q_{2}}^{(2)}(\alpha, \beta)= f_{w}\left[t_{p}, \frac{1}{6}\left(\alpha_{p-3}+4 \alpha_{p-2}+\alpha_{p-1}\right), \frac{1}{2}\left(\beta_{p-2}+\beta_{p-1}\right)\right] . \\
& \frac{1}{2}\left(\delta_{q_{2}}, p-2\right. \\
&
\end{aligned}
$$

Hence by means of the mean-value theorem we have

$$
\begin{aligned}
& \sum_{q_{1}}\left[J_{p q_{1}}^{(2)}(\alpha, \beta)-J_{p q_{1}}^{(2)}\left(\alpha^{\prime}, \beta^{\prime}\right)\right]^{2}+\sum_{q_{2}}\left[J_{p q_{2}}^{(2)}(\alpha, \beta)-J_{p q_{2}}^{(2)}\left(\alpha^{\prime}, \beta^{\prime}\right)\right]^{2} \\
& \quad \leq C^{2}\left[\left\{\left(\alpha_{p-3}-\alpha_{p-3}^{\prime}\right)^{2}+\left(\alpha_{p-2}-\alpha_{p-2}^{\prime}\right)^{2}+\left(\alpha_{p-1}-\alpha_{p-1}^{\prime}\right)^{2}\right\}\right. \\
& \left.\quad+\left\{\left(\beta_{p-2}-\beta_{p-2}^{\prime}\right)^{2}+\left(\beta_{p-1}-\beta_{p-1}^{\prime}\right)^{2}\right\}\right],
\end{aligned}
$$


where $C$ is positive constant such that

$$
\left|f_{x x}(t, x, w)\right|,\left|f_{x w}(t, x, w)\right|,\left|f_{w w}(t, x, w)\right| \leq C
$$

for all $(t, x, w) \in U$. By (36) and (37), we thus obtain

$$
\left|J_{h}(\alpha, \beta)-J_{h}\left(\alpha^{\prime}, \beta^{\prime}\right)\right| \leq M^{\prime}\left|\left(\alpha-\alpha^{\prime}, \beta-\beta^{\prime}\right)\right|
$$

where $M^{\prime}=\sqrt{3} C$. Clearly $M^{\prime}$ is independent of $h$.

\section{Existence of Spline Approximations}

First let us estimate $|F(\hat{\alpha}, \hat{\beta})|$. Since $\hat{w}_{h}(t)=\dot{\hat{x}}_{h}(t) \quad(0 \leq t \leq 1)$, we have

$$
F_{h}^{(1)}(\hat{\alpha}, \hat{\beta})=\left\{\frac{\hat{\alpha}_{p}-\hat{\alpha}_{p-1}}{h}-\hat{\beta}_{p}\right\}_{p=-2}^{n-1}=0
$$

Further, since

$$
\hat{x}_{h}\left(t_{p}\right)=\hat{x}\left(t_{p}\right), \quad \dot{\hat{x}}_{h}\left(t_{p}\right)=\dot{\hat{x}}\left(t_{p}\right) \quad(p=0, n)
$$

by (19), we have

$$
\begin{aligned}
F_{h}^{(3)}(\hat{\alpha}, \hat{\beta})= & \left\{\frac{1}{\sqrt{h}} A_{0} \hat{x}_{h}(0)-\frac{1}{\sqrt{h}} B_{0} \hat{w}_{h}(0)-\frac{a}{\sqrt{h}},\right. \\
& \left.\frac{1}{\sqrt{h}} A_{1} \hat{x}_{h}(1)+\frac{1}{\sqrt{h}} B_{1} \hat{w}_{h}(1)-\frac{b}{\sqrt{h}}\right\}=0 .
\end{aligned}
$$

For $F_{h}^{(2)}(\hat{\alpha}, \hat{\beta})$, by $(20)$ and $(21)$, we have

$$
\begin{aligned}
& F_{h}^{(2)}(\hat{\alpha}, \hat{\beta})=\left\{\dot{\hat{w}}_{h}\left(t_{p}\right)-f\left(t_{p}, \hat{x}_{h}\left(t_{p}\right), \hat{w}_{h}\left(t_{p}\right)\right)\right\}_{p=0}^{n} \\
& =\left\{\left[\dot{\hat{w}}_{h}\left(t_{p}\right)-\dot{\hat{w}}\left(t_{p}\right)\right]\right. \\
& \left.\quad+\left[f\left(t_{p}, \hat{x}\left(t_{p}\right), \hat{w}\left(t_{p}\right)\right)-f\left(t_{p}, \hat{x}_{h}\left(t_{p}\right), \hat{w}_{h}\left(t_{p}\right)\right)\right]\right\}_{p=0}^{n} \\
& =O\left(h^{2}\right)(h \rightarrow 0) .
\end{aligned}
$$

Therefore we have 


$$
\left|F_{h}^{(2)}(\hat{\alpha}, \hat{\beta})\right|=\sqrt{n+1} O\left(h^{2}\right)
$$

Since $n=\frac{1}{h}$, we then have

$$
\left|F_{h}^{(2)}(\hat{\alpha}, \hat{\beta})\right|=O\left(h^{\frac{3}{2}}\right)
$$

Thus we see that

$$
\left|F_{h}(\hat{\alpha}, \hat{\beta})\right| \leq L h^{\frac{3}{2}}
$$

for any $h \leq h_{1}$ provided $h_{1}$ is sufficiently small. Here clearly $L$ is a positive constant independent of $h$. Inequality (39) expresses that $(\alpha, \beta)=$ $(\hat{\alpha}, \hat{\beta})$ is an approximate solution of $F_{h}(\alpha, \beta)=0$. Hence we apply Proposition 2 of $[3]$ (p. 123) to the determining equation $F_{h}(\alpha, \beta)=0$ to prove the existence of its solution, that is, the existence of spline approximations to the solution of the problem (4)-(7). By (20) and (21), let us note that there is a positive constant $N$ such that

$$
\left\|\left(\hat{x}_{h}, \hat{w}_{h}\right)-(\hat{x}, \hat{w})\right\|_{\infty}=\sup _{0 \leq t \leq 1}\left|\left(\hat{x}_{h}(t), \hat{w}_{h}(t)\right)-(\hat{x}(t), \hat{w}(t))\right| \leq N h^{3}
$$

for any $h \leq h_{2}$ provided $h_{2}$ sufficiently small. We suppose that $N h_{2}^{3}<\delta$, and consider the set $V_{h}$ defined by

$$
V_{h}=\left\{(t, x, w)||(x, w)-\left(\hat{x}_{h}(t), \hat{w}_{h}(t)\right) \mid \leq \delta-N h^{3}, t \in[0,1]\right\} .
$$

Then by (40) it is clear that

$$
V_{h} \subset U \quad \text { for any } h \leq h_{2} .
$$

Let $\Omega_{h}$ be the set defined by

$$
\Omega_{h}=\left\{(\alpha, \beta)||(\alpha, \beta)-(\hat{\alpha}, \hat{\beta}) \mid \leq \delta-N h^{3}\right\},
$$

then for

$$
x_{h}(t)=\sum_{i p=-3}^{n-1} \alpha_{p} Q_{4}\left(\frac{t}{h}-p\right) \text { and } w_{h}(t)=\sum_{p=-2}^{n-1} \beta_{p} Q_{3}\left(\frac{t}{h}-p\right)
$$

with $(\alpha, \beta) \in \Omega_{h}$, by Lemma 4 we have 


$$
\left|x_{h}(t)-\hat{x}_{h}(t)\right|^{2}+\left|w_{h}(t)-\hat{w}_{h}(t)\right|^{2} \leq|\alpha-\hat{\alpha}|+|\beta-\hat{\beta}| \quad(0 \leq t \leq 1),
$$

consequently $\left(t, x_{h}(t), w_{h}(t)\right) \in V_{h} \subset U$ for any $t \in[0,1]$. This means that $F_{h}(\alpha, \beta)$ is defined on the region $\Omega_{h}$ for any $h \leq h_{2}$.

Now by (34) and (38) it holds that

$$
\left|J_{h}^{-1}(\hat{\alpha}, \hat{\beta})\right| \leq M \quad \text { for any } h \leq h_{0}
$$

and

$$
\begin{gathered}
\left|J_{h}(\alpha, \beta)-J_{h}(\hat{\alpha}, \hat{\beta})\right| \leq M^{\prime}|(\alpha, \beta)-(\hat{\alpha}, \hat{\beta})| \\
\text { for any } h \leq h_{2} \text { and any }(\alpha, \beta) \in \Omega_{h} .
\end{gathered}
$$

Take an arbitrary positive number $k<1$ and put $\delta_{1}=\min \left[\frac{k}{M M^{\prime}}, \delta-N h_{2}^{3}\right]$. If we take sufficiently small $h_{3} \leq \min \left(h_{0}, h_{1}, h_{2}\right)$, then it is possible to take $\delta_{h}$ so that

$$
\frac{M L h^{\frac{3}{2}}}{1-k} \leq \delta_{h} \leq \delta_{1} \quad \text { for any } h \leq h_{3} .
$$

Let $\Omega_{\delta_{h}}$ be the set defined by

$$
\Omega_{\delta_{h}}=\left\{(\alpha, \beta)||(\alpha, \beta)-(\hat{\alpha}, \hat{\beta}) \mid \leq \delta_{h}\right\}
$$

Then for any $h \leq h_{3}$ and any $(\alpha, \beta) \in \Omega_{\delta_{h}}$, we have $|(\alpha, \beta)-(\hat{\alpha}, \hat{\beta})| \leq \delta_{h} \leq \delta-N h_{2}^{3} \leq \delta-N h^{3}$, which means $(\alpha, \beta) \in \Omega_{h}$. Hence we see that

$$
\Omega_{\delta_{h}} \subset \Omega_{h} \quad \text { for any } h \leq h_{3} \text {. }
$$

Now for any $h \leq h_{3}$ and any $(\alpha, \beta) \in \Omega_{\delta_{h}}$, by (43) we have

(46) $\left|J_{h}(\alpha, \beta)-J_{h}(\hat{\alpha}, \hat{\beta})\right| \leq M^{\prime}|(\alpha, \beta)-(\hat{\alpha}, \hat{\beta})| \leq M^{\prime} \delta_{h} \leq M^{\prime} \delta_{1} \leq \frac{k}{M}$.

Moreover by (39) and (44) we have:

$$
\frac{M\left|F_{h}(\hat{\alpha}, \hat{\beta})\right|}{1-k} \leq \frac{M L h^{\frac{3}{2}}}{1-k} \leq \delta_{h} \quad \text { for any } h \leq h_{3} .
$$


The expressions (42), (45), (46) and (47) show that the conditions of Proposition 2 of $[3]$ (p. 123) are all fulfilled. Thus we see that the determining equation $F_{h}(\alpha, \beta)=0$ has one and only one solution $(\alpha, \beta)=$ $(\bar{\alpha}, \bar{\beta})$ in $\Omega_{\delta_{h}}$. This proves the existence of spline approximations to the solution of the problem (4)-(7).

\section{Convergence of Spline Approximations}

By Proposition 2 of $[3]$ (p. 123), the solution $(\bar{\alpha}, \bar{\beta})$ of the determining equation $F_{h}(\alpha, \beta)=0$ satisfies the inequality

$$
|(\bar{\alpha}, \bar{\beta})-(\hat{\alpha}, \hat{\beta})| \leq \frac{M L h^{\frac{3}{2}}}{1-k} \quad \text { for any } h \leq h_{3} .
$$

Let $\bar{\alpha}=\left(\bar{\alpha}_{-3}, \bar{\alpha}_{-2}, \ldots, \bar{\alpha}_{n-1}\right)$ and $\bar{\beta}=\left(\bar{\beta}_{-2}, \bar{\beta}_{-1}, \ldots, \bar{\beta}_{n-1}\right)$, and put

$$
\begin{aligned}
& \bar{x}_{h}(t)=\sum_{p=-3}^{n-1} \bar{\alpha}_{p} Q_{4}\left(\frac{t}{h}-p\right), \\
& \bar{w}_{h}(t)=\sum_{p=-2}^{n-1} \bar{\beta}_{p} Q_{3}\left(\frac{t}{h}-p\right) .
\end{aligned}
$$

Since $F_{h}^{(1)}(\bar{\alpha}, \bar{\beta})=0$, it is clear that

$$
\dot{\bar{x}}_{h}(t)=\bar{w}_{h}(t) \quad(0 \leq t \leq 1)
$$

From (48) follows

$$
|\bar{\alpha}-\hat{\alpha}|,|\bar{\beta}-\hat{\beta}|=O\left(h^{\frac{3}{2}}\right) \quad(h \rightarrow 0) .
$$

Therefore by Lemma 3 and 2 we have

$$
\left\|\bar{x}_{h}-\hat{x}_{h}\right\|,\left\|\bar{w}_{h}-\hat{w}_{h}\right\|=O\left(h^{2}\right) \quad(h \rightarrow 0),
$$

and by Lemma 4 we have

$$
\left\|\bar{x}_{h}-\hat{x}_{h}\right\|_{\infty},\left\|\bar{w}_{h}-\hat{w}_{h}\right\|_{\infty}=O\left(h^{\frac{3}{2}}\right) \quad(h \rightarrow 0) .
$$

On the other hand,

$$
\left\|\hat{x}_{h}-\hat{x}\right\|_{\infty}=O\left(h^{4}\right) \quad(h \rightarrow 0)
$$


by (20), and

$$
\left\|\hat{w}_{h}-\hat{w}\right\|_{\infty}=O\left(h^{3}\right) \quad(h \rightarrow 0)
$$

by (21). Hence from (50) and (51) we have:

$$
\left\|\bar{x}_{h}-\hat{x}\right\|_{1}, \| \bar{w}_{h}-\hat{w}||=O\left(h^{2}\right) \quad(h \rightarrow 0)
$$

and

$$
\left\|\bar{x}_{h}-\hat{x}\right\|_{\infty},\left\|\bar{w}_{h}-\hat{w}\right\|_{\infty}=O\left(h^{\frac{3}{2}}\right) \quad(h \rightarrow 0) .
$$

Since $\dot{\bar{x}}_{h}(t)=\bar{w}_{h}(t)(0 \leq t \leq 1)$ by $(49)$ and $\hat{w}(t)=\dot{\hat{x}}(t)$ by the definition, we thus have

Theorem. In a sufficiently small neighborhood of the isolated solution $\hat{x}(t)$ of the problem (1)-(3), there is a cubic spline function $\bar{x}_{h}(t)$ of the form

$$
\bar{x}_{h}(t)=\sum_{p=-3}^{n-1} \bar{\alpha}_{p} Q_{4}\left(\frac{t}{h}-p\right)
$$

such that

(i) the coefficient $\bar{\alpha}=\left(\bar{\alpha}_{-3}, \bar{\alpha}_{-2}, \ldots, \bar{\alpha}_{n-1}\right)$ satisfies the determining equation $F_{h}(\alpha, \beta)=0$ together with the coefficient $\bar{\beta}=\left(\bar{\beta}_{-2}, \bar{\beta}_{-1}, \ldots, \bar{\beta}_{n-1}\right)$ of the quadratic function defined by

$$
\bar{w}_{h}(t)=\dot{\bar{x}}_{h}(t)=\sum_{p=-2}^{n-1} \bar{\beta}_{p} Q_{3}\left(\frac{t}{h}-p\right) \quad(0 \leq t \leq 1),
$$

(ii)

$$
\left\|\bar{x}_{h}-\hat{x}\right\|,\left\|\dot{\bar{x}}_{h}-\dot{\hat{x}}_{\|}\right\|=O\left(h^{2}\right) \quad(h \rightarrow 0)
$$

and

$$
\left\|\bar{x}_{h}-\hat{x}_{\|}\right\|_{\infty},\left\|\dot{\bar{x}}_{h}-\dot{\hat{x}}\right\|_{\infty}=O\left(h^{\frac{3}{2}}\right) \quad(h \rightarrow 0) .
$$

The expression (53) clearly implies the uniform convergence of $\bar{x}_{h}(t)$ to the exact solution $\hat{x}(t)$ on $[0,1]$ together with its first order derivative.

Remark. If $B_{0}=0$, then $\bar{x}_{h}(0)=\hat{x}(0)$ by (14). Then applying 
Schwarz's inequality to the equality

$$
\bar{x}_{h}(t)-\hat{x}(t)=\int_{0}^{1}\left[\dot{\bar{x}}_{h}(s)-\dot{\hat{x}}(s)\right] d s,
$$

we have

$$
\left|\bar{x}_{h}(t)-\hat{x}(t)\right| \leq\left\|\dot{\bar{x}}_{h}-\dot{\hat{x}}\right\|=O\left(h^{2}\right) \quad(0 \leq t \leq 1),
$$

that is,

$$
\left\|\bar{x}_{h}-\hat{x}\right\|_{\infty}=O\left(h^{2}\right) \quad(h \rightarrow 0) .
$$

One can see in a similar way equality (54) is valid also when $B_{1}=0$. Thus if $B_{0}=0$ or $B_{1}=0$, we always have (54).

\section{Acknowledgments}

The author wishes to express his gratitude to Professor M. Urabe for many helpful discussions, and for his careful reading of the manuscript. Thanks are also due to Professor S. Huzino for suggesting the problem of spline approximations to the two-point boundary value problems.

\section{References}

[1] Ahlberg, J.H., E.N. Nilson and J.L. Walsh, The Theory of Splines and Their Applications, Academic Press, New York, 1967.

[2] Bickley, W.G., Piecewise cubic interpolation and two-point boundary problems, Comput. J. 11 (1968), 206-208.

[3] Urabe, M., Galerkin's procedure for nonlinear periodic systems, Arch. Rational Mech. Anal. 20 (1965), 120-152.

[4] - An existence theorem for multi-point boundary value problems, Funkcial. Ekvac. 9 (1966), 43-60.

[5] - Numerical solution of multi-point boundary value problems in Chebyshev series — theory of the method, Numer. Math. 9 (1967), 341-366. 